\section{Membranous nephropathy complicating relapsing polychondritis: A case report}

\author{
Christopher Rice ${ }^{1,2}$, Vatsalya Kosuru2,3, John Jason White ${ }^{2,3}$, \\ Christine Van Beek ${ }^{4}$, Rachel Elam ${ }^{1,2 *}$, Michael Clemenshaw ${ }^{5}$, Laura \\ Carbone $^{1,2}$ and Leighton James ${ }^{3}$
}

1'Division of Rheumatology, Medical College of Georgia, Augusta University, GA, USA

${ }^{2}$ Charlie Norwood Veterans Affairs Medical Center, Augusta, GA, USA

${ }^{3}$ Division of Nephrology, Medical College of Georgia, Augusta University, GA, USA

${ }^{4}$ Ameri Path, 225 N.E. 97th St, Suite 600, Oklahoma City, OK, USA

${ }^{5}$ Nuclear Medicine Section, Department of Radiology and Imaging, Medical College of Georgia,

Augusta University, GA, USA

\section{Abstract}

Background: Relapsing polychondritis is a rare systemic disease characterized by recurrent inflammation, and often destruction, of cartilaginous tissues. Renal manifestations are rare. Membranous nephropathy complicating relapsing polychondritis has been reported only once previously, and there is no standardized treatment for membranous nephropathy associated with relapsing polychondritis.

Case presentation: A 67-year-old Caucasian man with a history of chronic renal disease presented with 9 months of progressive dyspnea on exertion and 5 months of erythema, pain, and collapse of auricular cartilage. Imaging studies confirmed active inflammation of laryngeal, auricular, and costal cartilage and he was diagnosed with relapsing polychondritis. Patient had longstanding proteinuria and renal biopsy demonstrated membranous nephropathy. Patient initially showed renal and respiratory improvement with etanercept, a tumor necrosis factor alpha inhibitor, treatment. However, subsequent disease and treatment-related complications led to a progressive overall clinical decline and patient died approximately 1 year following relapsing polychondritis diagnosis.

Conclusion: Membranous nephropathy may rarely complicate relapsing polychondritis. In our case, both the cartilaginous inflammation and the renal disease improved after treatment with tumor necrosis factor alpha blockade, however complications of existing airway disease led to recurrent hospitalizations and eventually death.

\author{
More Information \\ *Address for Correspondence: Rachel Elam, \\ MD, ScM, Augusta University Department of \\ Medicine, Division of Rheumatology, 1120 15th \\ Street Augusta, GA 30912, USA, Tel: 706-721- \\ 0395, Fax: 706-721-1402, \\ Email: relam@augusta.edu \\ Submitted: June 07, 2021 \\ Approved: October 06, 2021 \\ Published: October 07, 2021 \\ How to cite this article: Rice C, Kosuru V, \\ White JJ, Beek CV, Elam R, et al. Membranous \\ nephropathy complicating relapsing \\ polychondritis: A case report. J Clini Nephrol. \\ 2021; 5: 084-087. \\ DOI: 10.29328/journal.jcn.1001080 \\ Copyright: (c) 2021 Rice C, et al. This is an \\ open access article distributed under the \\ Creative Commons Attribution License, which \\ permits unrestricted use, distribution, and \\ reproduction in any medium, provided the \\ original work is properly cited. \\ Keywords: Relapsing polychondritis; \\ Membranous nephropathy; Systemic vasculitis; \\ Tumor necrosis factor alpha inhibitor; Case \\ report \\ Check for updates \\ OPEN ACCESS
}

\section{Introduction}

Relapsing polychondritis (RP) is an uncommon systemic disease characterized by recurrent inflammation, and often destruction, of cartilaginous tissues. RP most commonly involves the ears, nose, eyes, joints and respiratory tract. Renal disease occurs in less than

$10 \%$ of RP cases, but confers a worse prognosis when present [1]. The most common renal pathological findings include mesangial expansion with cell proliferation and necrotizing segmental glomerulonephritis with crescents; however, various other presentations have also been identified. In a series of 29 patients with RP from Mayo
Clinic, 22\% had any evidence of renal involvement defined as hematuria or proteinuria. Of these patients, 11 had biopsy-proven renal involvement, with reported lesions including mesangial expansion, tubulointerstitial nephritis, and segmental necrotizing crescentic glomerulonephritis [2]. In this series, RP patients with renal disease were older and more frequently developed arthritis and extrarenal vasculitis than those without renal disease [2]. Furthermore, IgA nephropathy has also been described in association with RP $[3,4]$. The etiology of renal disease associated with $\mathrm{RP}$ remains unknown. The presence of granular deposition of IgG, IgA, IgM and complements on immunofluorescence study of renal biopsies in RP suggests that the deposition of 
immune complexes may play a role in the pathogenesis of glomerular lesions $[5,6]$.

To our knowledge, there is only one previous case report of membranous nephropathy (MN) complicating RP [7]. We describe a second case of a MN occurring as a manifestation of RP in a patient with widespread disease. Both the renal and extra-renal RP manifestations improved after tumor necrosis factor (TFN) alpha blockade.

\section{Case presentation}

A 67-year-old Caucasian man presented with a ninemonth history of dyspnea on exertion accompanied by a nonproductive cough. He had a five-month history of pain, erythema, and warmth on the apical helices of both ears associated with changes in the appearance of the bridge of his nose. He had recently noted symmetric decreased sensation in his lower extremities, arthralgias, and a 23-pound unintentional weight loss. Past medical history was remarkable for a ten-year history of nephrotic range proteinuria with chronic kidney disease (CKD) and multiple episodes of acute renal insufficiency for which he had previously declined renal biopsy.

On physical examination, vital signs revealed a heart rate of 81 beats per minute, 19 respirations per minute, blood pressure of 150/89, and a temperature of 98.1 degrees Fahrenheit. There were bilateral cauliflower ear deformities, left worse than right, and a saddle nose deformity. Rhonchi were diffusely present and skin examination was notable for vitiligo. Neurological examination showed decreased sensation in his hands and his lower body distal to the umbilicus with normal strength. There was no active synovitis.

Laboratory studies were significant for anemia of chronic disease with a hemoglobin of $7 \mathrm{~g} / \mathrm{dL}$, albumin $3.1 \mathrm{~g} / \mathrm{dL}$, total protein $6.0 \mathrm{~g} / \mathrm{dL}$ and elevated inflammatory markers (ESR $>100 \mathrm{~mm} / \mathrm{hr}$ and CRP of $26 \mathrm{mg} / \mathrm{l}$ ). Serologies revealed a negative antinuclear antibody, anti-neutrophil cytoplasmic antibody, Anti-Ro antibody, Anti-La antibody, and double stranded DNA antibody. Serum protein electrophoresis showed no monoclonal M spike. Cryoglobulins and type 2 collagen antibodies were not present. Computed tomography of the thorax revealed bronchial thickening and ground glass nodules with upper lobe predominance. Pulmonary function tests were significant for severe airflow obstruction not responsive to bronchodilators with a total lung capacity of $85 \%$, a diffusion capacity of the lungs for carbon monoxide (DLCO) of 60\% and severe obstruction in the flow volume loop. Bronchoscopy was significant for severe bronchomalacia of the right and left proximal main stem airway with complete collapse during expiration. F-18 Fluorodeoxyglucose (FDG) Positron Emission Tomography - Computed Tomography (PET-CT) scan showed anterior tracheal wall and main stem bronchial wall thickening with severe luminal narrowing.
There was diffuse increased inflammatory FDG uptake in the cartilaginous tracheal rings, costal cartilages and auricles (Figure 1).

A sural nerve biopsy showed severe axonal neuropathy with associated myelin loss without active vasculitis seen. An ophthalmology exam revealed clinically significant bilateral cataracts but otherwise no uveitis, scleritis, or any retinal changes.

For the last 10 years, he had renal disease with a stable creatinine $1.1 \mathrm{mg} / \mathrm{dL}$, intermittent urinalysis with protein $>500 \mathrm{mg} / \mathrm{dL}(4+)$ and trace microhematuria, and proteinuria of $3.39 \mathrm{~g}$ in 24 hours but had declined renal biopsy. However, he now had persistent 4+ proteinuria on multiple urinalysis and agreed to undergo a renal biopsy. The renal biopsy revealed membranous nephropathy with associated segmental sclerosis and moderate interstitial fibrosis (30$40 \%$ ). The light microscopy with methenamine-silver stain showed preserved glomerular architecture with thickened capillary walls and open capillary loops. Immunofluorescence showed glomerular capillary wall staining for $\operatorname{IgG}$, IgA (trace), IgM, C3 (trace), kappa, and lambda in a granular pattern. Electron microscopy demonstrated electron-dense deposits distributed irregularly in the sub-epithelial portion of glomerular basement membrane and effacement of foot processes (Figure 2). Phospholipase A2 Receptor (PLA2R) staining of these glomerular sub-epithelial deposits was negative.

He was diagnosed with RP and underwent airway stenting for symptom management. Unfortunately, this was complicated by laceration of the left main stem bronchus after Y-stent deployment. Antibiotics were administered including vancomycin, cefepime, and metronidazole for related aspiration pneumonia. Following resolution of this, he was treated with etanercept, a tumor necrosis factor (TNF) alpha inhibitor, at a dose of $50 \mathrm{mg}$ subcutaneous every 7 days. His arthralgia and dyspnea improved markedly, and
A.

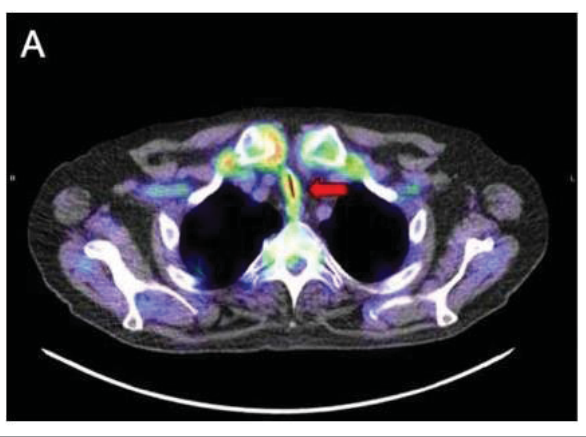

B.

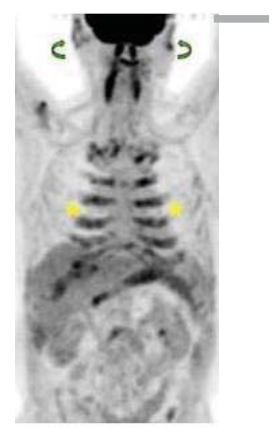

Figure 1: F-18 Fluorodeoxyglucose (FDG) Positron Emission Tomography-Computed Tomography Imaging Demonstrating Diffuse Cartilaginous Inflammatory FDG Uptake. A. Axial fused Positron Emission Tomography-Computed Tomography (PET-CT): Anterior tracheal wall thickening with severe luminal narrowing and diffuse increased FDG uptake (red arrow). B. Anterior Whole Body Maximum Intensity Projection FDG PET-CT Image: Diffuse increased FDG uptake in the costal cartilage (yellow stars) and auricles (green curved arrows). 


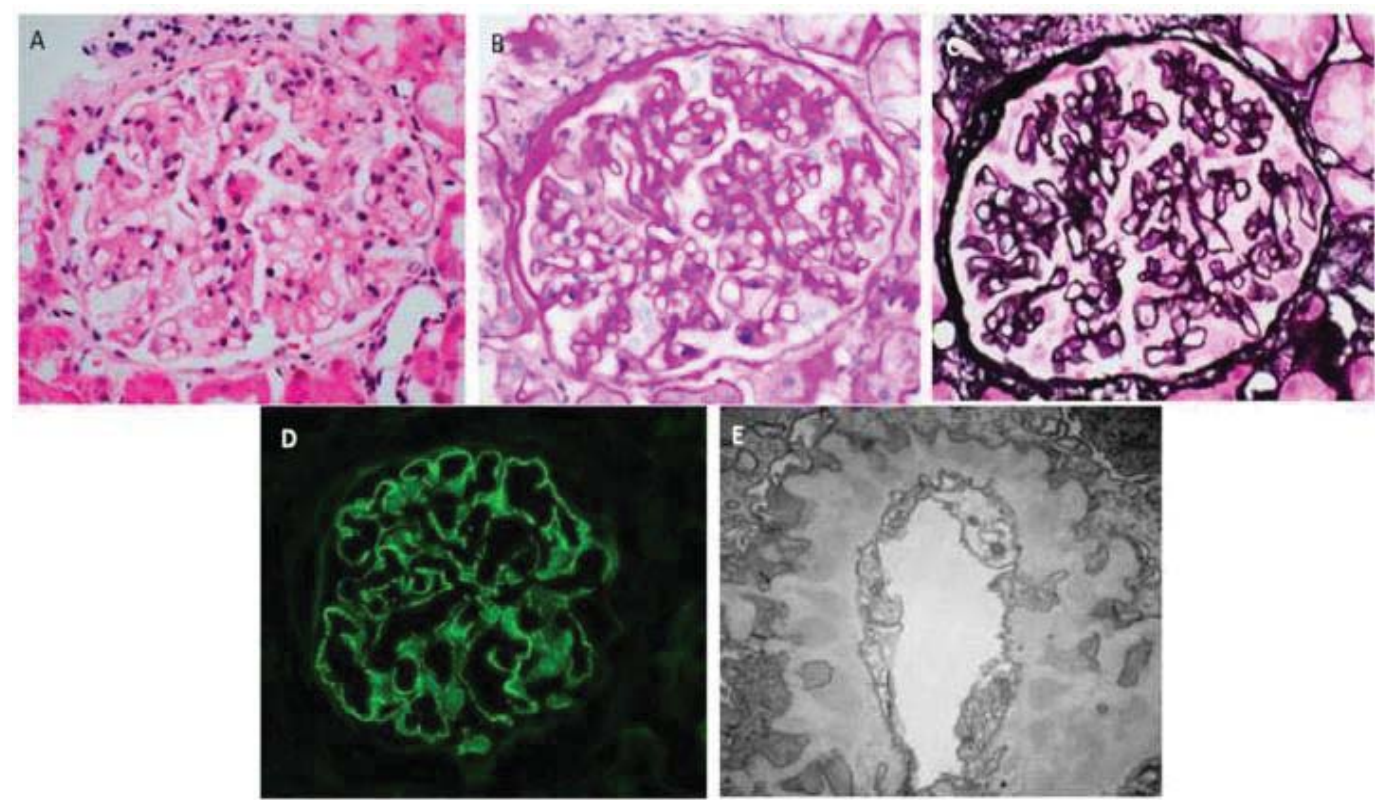

Figure 2: Kidney Biopsy Demonstrating Membranous Nephropathy. A. Hematoxylin and eosin stain (original magnification x400): Normocellular glomerulus. B. Periodic acid-Schiff stain (original magnification x400): Image shows a glomerulus with slight mesangial prominence and irregular capillary wall thickening. There is no significant hypercellularity. C. Jones methenamine silver stain (original magnification $\times 400$ ): Holes and occasional spikes are visible along glomerular capillary basement membranes. D. IgG immunofluorescence (original magnification $\times 400$ ): $\lg$ is positive in glomerular capillary walls in a granular pattern. E. Electron microscopy (original magnification x20,000): There are continuous subepithelial deposits along glomerular capillary walls, which are well-incorporated into basement membranes and separated or occasionally surrounded by projections of basement membrane material consistent with Churg and Ehrenreich ultrastructural stage II to III membranous nephropathy [8]

repeat airway stenting was not needed due to improvement in breathing status. Renal function remained stable ( $\mathrm{Cr} 1.0$ $1.1 \mathrm{mg} / \mathrm{dL}$ ) and the proteinuria improved to sub-nephrotic range (193 mg/24 hr). Multiple subsequent urinalysis showed protein ranging from none to $30 \mathrm{mg} / \mathrm{dL}(1+)$.

Unfortunately, the patient's overall clinical status progressively declined, at least in part from complications and treatment of RP. Patient had multiple hospitalizations for infection, including complicated urinary tract infections and chronic prostatitis as well as refractory Clostridium difficile colitis necessitating interruptions in etanercept therapy. His peripheral neuropathy resulted in frequent falls and he ultimately suffered a hip fracture. Approximately 14 months after initial diagnosis and treatment he was admitted for hypoxic and hypercapneic respiratory failure due to staphylococcal pneumonia. Bronchoscopy revealed no significant change in expiratory airway collapse or active upper airway inflammation and his condition was too poor for repeat PET-CT evaluation of disease activity. Repeat airway stenting was attempted as a supportive measure but did not allow successful weaning from mechanical ventilation. Patient died during this admission.

\section{Discussion}

To our knowledge, there is only one previous case report of MN complicating RP [7]. Similar to our case, this patient also originally declined renal biopsy and the proteinuria was attributed to diabetes. In our patient's case, the longstanding proteinuria had been attributed to hypertension. Proteinuria in the range of $0.5-1 \mathrm{~g}$ daily is seen in patients diagnosed with essential hypertension; however, nephrotic-rangeproteinuria is quite unusual with hypertensive nephrosclerosis. With the exception of malignant hypertension, in cases where nephrotic-range proteinuria is encountered in patients diagnosed with essential hypertension, other underlying glomerular pathologies should be considered. The subsequent results of renal biopsy from the case in the literature, like our case, showed evidence of MN with sub-epithelial electrondense deposits, which explains the finding of nephroticrange proteinuria. In contrast to this prior report, in which the patient was treated with prednisone and cyclosporine [7], we elected to use etanercept for treatment of his RP including the renal manifestations. TNF alpha blockers, including etanercept, can be used to treat RP [9], usually in combination with corticosteroids [10].

There is no established therapy for treatment of RP. Therapeutic approaches have included use of nonsteroidal anti-inflammatory drugs (NSAIDs) for pain, systemic corticosteroids for NSAID-resistance RP, and immunosuppressive agents in organ or life-threatening, corticosteroid-intolerant or corticosteroid-dependent disease [5]. We decided not to use corticosteroids because of the recent pulmonary infection and the patient's concern for increased risk for the novel Coronavirus Disease 2019 (COVID-19). Interestingly, TNF alpha blockers can rarely cause glomerulonephritis with various reported manifestations: membranous nephropathy, crescentic necrotizing glomerulonephritis, extra-capillary glomerulonephritis, and minimal change disease. These cas- 
es are reported in the context of use of etanercept for rheumatoid arthritis, psoriasis, juvenile psoriatic arthritis and ankylosing spondylitis [11-13]. However, this association has not been described in the setting of RP.

We acknowledge that the exact relationship between the $\mathrm{MN}$ and relapsing polychondritis is not clear in this case. The presence of intermittent heavy proteinuria on urinalysis assessments for several years before the onset of RP, raises the possibility that the RP and MN may be two separate diseases. RP patients may have a genetic predisposition for autoimmunity, and a subset of these patients develop an overlapping autoimmune disease such as autoimmune thyroid disease, autoimmune hemolytic anemia, and systemic lupus erythematosus, among others [14]. Perhaps MN is another autoimmune condition that could be added to this list. However, the substantial reduction in proteinuria after treatment with etanercept could be a clue to a possible association between the RP and MN. We did not have serial 24-hour urine studies and subsequent urinalyses were confounded by frequent presentations with simultaneous urinary tract infections. Despite these limitations, further urinalyses continue to show only trace to $1+$ proteinuria after starting RP-directed therapy with etanercept as opposed to $4+$ proteinuria previously. This temporal association of treatment with etanercept, improvement of RP symptoms, and the improvement of proteinuria suggests that MN could be secondary to RP. The MN may have presented as the initial manifestation of RP. A similar pattern can occur in membranous lupus nephritis, where the renal disease can precede the development of other lupus symptoms [15]. Regardless, it is noteworthy that patient's proteinuria secondary to MN improved with etanercept whether or not the renal disease was an associated or independent process from RP. The potential efficacy for TNF alpha blockade in reducing proteinuria in $\mathrm{MN}$ has been reported in a case of MN in the setting of psoriasis [16], and this case lends additional evidence for TNF alpha blocking therapy in MN. We also cannot exclude the possibility that the patient was already incidentally entering early remission of $\mathrm{MN}$ at the time etanercept was initiated, given that there were no recent appearing (stage I) deposits by electron microscopy.

Another limitation was incomplete knowledge of patient's RP disease activity at the time of his death. Although conventional CT and repeat bronchoscopy with biopsy showed no continued airway inflammation, repeat PET-CT imaging was not obtained for direct assessment of active inflammation due to critical illness. However, even in the absence of active RP at his time of death, we suspect the extensive complications from his RP course contributed to his death despite treatment with etanercept.

We suggest that renal status should be carefully investigated in patients with RP and renal disease, as it may potentially be an early manifestation of RP and may respond to TNF alpha blockade.

\section{References}

1. Gergely P Jr, Poór G. Relapsing polychondritis. Best Pract Res Clin Rheumatol. 2004; 18: 723-738.

PubMed: https://pubmed.ncbi.nlm.nih.gov/15454129/

2. Chang-Miller A, Okamura M, Torres VE, Michet CJ, Wagoner RD, et al. Renal involvement in relapsing polychondritis. Medicine. 1987; 66: 202-217.

PubMed: https://pubmed.ncbi.nlm.nih.gov/3574118/

3. Dalal BI, Wallace AC, Slinger RP. IgA nephropathy in relapsing polychondritis. Pathology. 1988; 20: 85-89.

PubMed: https://pubmed.ncbi.nlm.nih.gov/3287308/

4. Meignan F, Maillefert F, Bargues L, Colle B, Colon S. Association of glomerular nephropathy with IgA deposits and relapsing polychondritis. Ann Med Interne (Paris). 1992; 143: 548-550.

PubMed: https://pubmed.ncbi.nlm.nih.gov/1303599/

5. Borgia F, Giuffrida R, Guarneri F, Cannavò SP. Relapsing Polychondritis: An Updated Review. Biomedicines. 2018; 6: 84. PubMed: https://pubmed.ncbi.nlm.nih.gov/30072598/

6. Espinoza LR, Richman A, Bocanegra T, Pina I, Vasey FB, Rifkin SI, et al. Immune complex-mediated renal involvement in relapsing polychondritis. Am J Med. 1981: 71; 181-183.

PubMed: https://pubmed.ncbi.nlm.nih.gov/6454344/

7. Lee JE, Lee EK. A case of membranous nephropathy associated with relapsing polychondritis. Kidney Res Clin Pract. 2012; 31: 253-256. PubMed: https://pubmed.ncbi.nlm.nih.gov/26894028/

8. Churg J, Ehrenreich T. Membranous nephropathy. Perspect Nephrol Hypertens. 1973; 1: 443-448.

PubMed: https://pubmed.ncbi.nlm.nih.gov/4536436/

9. Moulis G, Sailler L, Pugnet G, Astudillo L, Arlet P. Biologics in relapsing polychondritis: a case series. Clin Exp Rheumatol. 2013; 31: 937-939. PubMed: https://pubmed.ncbi.nlm.nih.gov/24021708/

10. Moulis G, PugnetG, Costedoat-Chalumeau N, MathianA, LerouxG, etal. Efficacy and safety of biologics in relapsing polychondritis: a French national multicentre study. Ann Rheuma Dis. 2018; 77: 1172-1178. PubMed: https://pubmed.ncbi.nlm.nih.gov/29535124/

11. Kaushik $P$, Rahmani M, Ellison W. Membranous glomerulonephritis with the use of etanercept in ankylosing spondylitis. Ann Pharmacother. 2011; 45: e62.

PubMed: https://pubmed.ncbi.nlm.nih.gov/22116994/

12. Ammar A, Zafar Ahmed Mahmood H, Shahid Z, Jain R, Chen G. Etanercept-associated Nephropathy. Cureus. 2019; 11: e5419. PubMed: https://pubmed.ncbi.nlm.nih.gov/31632872/

13. Stokes MB, Foster K, Markowitz GS, Ebrahimi F, Hines W, et al. Development of glomerulonephritis during anti-TNF-alpha therapy for rheumatoid arthritis. Nephrol Dial Transplant. 2005; 20: 1400-1406. PubMed: https://pubmed.ncbi.nlm.nih.gov/15840673/

14. Cantarini L, Vitale A, Brizi MG, Caso F, Frediani B, et al. Diagnosis and classification of relapsing polychondritis. J Autoimmun. 2014; 4849: 53-59.

PubMed: https://pubmed.ncbi.nlm.nih.gov/24461536/

15. Yamada T, Itagaki F, Aratani S, Kawasaki S, Terada K, et al. A case of membranous nephropathy diagnosed with lupus nephritis 11 years after onset. CEN Case Rep. 2019; 8: 301-307. PubMed: https://pubmed.ncbi.nlm.nih.gov/31399881/

16. Santoro D, Postorino A, Costantino G, Savica V, Bellinghieri G. AntiTNF-alpha therapy in membranous glomerulonephritis. Clin Kidney J. 2012; 5: 487-488.

PubMed: https://pubmed.ncbi.nlm.nih.gov/26019836/ 